\title{
CHIRAL SYMMETRY BREAKING IN CRYSTAL GROWTH IS HYDRODYNAMIC CONVECTION RELEVANT?
}

\author{
B. Martin, A. Tharrington, and X-l. Wu \\ Department of Physics, University of Pittsburgh, Pittsburgh, PA 15260
}

\section{ABSTRACT}

The effects of mechanical stirring on nucleation and chiral symmetry breaking have been investigated for a simple inorganic molecule, sodium chlorate $\left(\mathrm{NaClO}_{3}\right)$. In contrast to earlier findings, our experiment suggests that the symmetry breaking may have little to do with hydrodynamic convection. Rather the effect can be reasonably accounted for by mechanical damage to incipient crystals. The catastrophic events, creating numerous small "secondary" crystals, produce statistical domination of one chiral species over the other. Our conclusion is supported by a number of observations using different mixing mechanisms.

\section{INTRODUCTION}

Question about origins, whether of the Cniverse, of the Earth or of life, have always held great fascination. One intriguing problem of this type is to explain chiral symmetry-breaking in nature; how for example, it came to be that nearly all the optically active amino acids of biological significance are levo- rather than dextro-rotatory. One of the compelling explanations is the process of auto-catalysis: the enhancement of the rate of growth of a population as that population increases. In the early 90 's auto-catalysis was demonstrated for the first time by Kondepudi et al. ${ }^{1}$ on crystalization of an inorganic salt in the presence of a hydrodynamic flow. In a simple, yet elegant experiment Kondepudi obtained some interesting data on the distribution of optical activity among crystals precipitating from a supersaturated solution of sodium chlorate under different mixing conditions. The handedness of the crystals was established visually, using crossed polarizers.

What Kondepudi established is the following: (1) When the solution was not stirred, of 1,000 crystals collected from 17 different crystalization, 525 were of levo (left-handed) and 475 of dextro (right-handed) chirality; that is, the total numbers were statistically equal, as were the numbers in each individual experiment. (2) When the solution was stirred at 100 r.p.m. during the crystalization process, the total numbers for the 11,829 crystals were consistent with a 50-50 distribution of D- and L-crystals. One exception, however, was that each of the the 32 individual crystalization gave rise to a set of crystals that were more than $99 \%$ levoor $99 \%$ dextro-rotatory.

This abservation is remarkable in that it suggests that the system undergoes a transition from a totally symmetric (achiral) state to a totally asymmetric (chiral) state simply by introducing a hydrodynamic flow, without careful control of temperature and other conditions. The authors attribute the observed selectivity to an autocatalytic effect arising from the rapid production of secondary nuclei from a single primary nucleus in the stirred system. Namely, the first primary nucleus (or micro-crystal) serves as a chiral template which is convected by the flow, triggering secondary nucleations that have the same chirality as the primary one.

Kondepudi's work has stimulated considerable interest in the scientific community. Metcalfe and Ottino have modeled the experiments by a simple autocatalytic reaction scheme combined with a chaotic mixing flow ${ }^{2}$. Due to repetitive stretching and folding of fluid elements chirality initially associated with an incipient seed spreads rapidly throughout the entire system. The symmetry breaking was also considered as one of many examples of imperfect mixing in autocatalytic chemical and biological systems ${ }^{3}$.

Kondepudi's experiment touches upon a number of issues including nucleation, convective diffusion, chaotic mixing, and autocatalysis. All of these are of considerable current interest. However, the experiment also raises many questions that need to be answered before the effect can rest on firm ground. The most pressing question is the physical origin of the secondary nucleation and its relationship to hydrodynamic convection. For this reason we have designed and carried out a series of experiments aimed at better control of interactions between the flow and the precipitated micro-crystals. Two flow geometries are used, mechanical stirring and electro-convection. In the mechanical-stirring experiments each sample contains approximately a single vortex and the flow is chaotic even at low stirring rate. The simple mechanical setup allows mass production of crystals under nearly identical conditions, ensuring adequate counting-statistics. On the other hand, in the electro-convection experiments, large numbers of vortices are generated and the flow characteristics can be tuned continuously. A critical finding of our experiment is that the degree of chiral symmetry breaking depends on the location as well as the speed of the magnetic mixer. In electro-convection, which has no mechanical moving part, there is no chiral symmetry breaking. Our experiment, therefore, suggests that mechanical damage to incipient crystals, rather than hydrodynamic flow, is responsible for the chiral symmetry breaking. 


\section{EXPERIMENTAL}

The crystalization experiments were performed using a $\mathrm{NaClO}_{3}$ solution with a weight fraction of $50 \%{ }^{4}$. The $\mathrm{NaClO}_{3}$ was first dissolved in distilled water and constantly stirred at room temperature for 8 hours to ensure complete dissolution of the solute. The solutions were then filtered through a $0.2 \mu \mathrm{m}$ filter before usage. The crystal growth was initiated by slow evaporation. At the given concentration, the nucleation occurs approximately 46 hours after the samples were prepared, and this time interval appears to be independent of the flow rate. In the experiments the crystals were grown to an average size of $\sim 1 \mathrm{~mm}$; this takes an additional a couple of hours for quiescent samples and less than an hour for stirred samples.

Crystals of different chiralities can be distinguished by their optical activity. A right-handed crystal will rotate polarized light clockwise while a left-handed crystal will rotate light counter-clockwise. A longworking-distance microscope with a pair of polarizers allows us to visually determine the handedness of the crystals even when they are only $\sim 100 \mu \mathrm{m}$ in size. Under the transmission of a white light source, the two different crystal species appear blue and brown by slightly un-crossing the polarizers a few degrees. Since in some cases thousands of crystals were produced in a single sample, only a representative region of a few hundred crystals were analyzed.

Two different flow geometries were employed in the experiments. In the first experiment, $\mathrm{NaClO}_{3}$ solution was contained in a cylindrical dish of $\sim 5 \mathrm{~cm}$ in diameter. A small Teflon coated magnetic mixer (1 $\mathrm{cm}$ long and $0.2 \mathrm{~cm}$ diameter) was used to stir the solution. The light weight of the magnetic mixer and the large surface tension of the solution kept the mixer floating on the liquid-vapor interface while driven by a horse-shoe magnet. The field gradient of the horse-shoe magnet kept the mixer centered on the interface, providing effective mixing near the surface as well as in the interior of the solution. To improve statistics, twenty identical samples were run simultaneously with the horse-shoe magnets connected in tandem, driven by a single DC motor. Flow visualization using Kalliroscopic fluid indicated that flow was spatiotemporally chaotic even at a very low stirring rate, $\sim 1 / 30 \mathrm{hz}$. In this experiment, the stirring rate $f$ as well as the volume of the solution $V$ are the control parameters. As we shall see, the volume $V$, which essentially determines the distance between the stirrer and the bottom of the dish, plays an important role for the outcomes of the experiment.

In the second experiment, flow was produced by electro-convection. In this geometry all the moving parts were eliminated, preventing mechanical damage to the precipitated crystals. The flow cell has a dimension of $12 \times 20 \mathrm{~cm}^{2}$ with a biased ac current flowing along the long dimension of the cell. The electrodes are made of platinum to reduce contamination of the solution. Directly underneath the cell is an array of 400 cylindrical magnets each having a field of $\sim 0.4 \mathrm{~T}$ near the surface of the magnets. The magnets are arranged in a square lattice with alternating poles pointing vertically. When a current passes through a thin layer of $\mathrm{NaClO}_{3}$ solution, an array of counter rotating vortices are formed. Depending on the strength of the current, both localized and delocalized vortices can be generated. Since the thickness of the fluid is small, $\sim 3 \mathrm{~mm}$, compared to the width of the cell, the flow is approximately two dimensional.

\section{EXPERIMENTAL OBSERVATION}

\section{Nucleation in Quiescent Solutions}

To ascertain that nucleation behaves normally with no substantial bias towards either species, a set of quiescent measurements were carried out. Here, our finding was rather similar to that of hipping and Pope ${ }^{5}$, and Kondepudi ${ }^{1}$. In a total of 5395 crystals obtained from 94 crystalization, 2523 were right handed and 2872 were left handed. The difference between the two species is about $6 \%$. We noted that similar bias was also observed in Kondepudi's experiment at the level of $\sim 10 \%$. If an order parameter is defined as $O=\left(N^{L}-N^{R}\right) /\left(N^{L}+N^{R}\right)$, we can plot the distribution function $P(O)$ for all 94 runs. Figure 1 shows $P(O)$ for the quiescent samples $(f=0 \mathrm{hz})$. For a comparison a binomial distribution with the width comparable to our measurements is also shown by the solid line. The bias (6\%) towards left-handed crystals, as illustrated in the graph, is statistically significant since the standard deviation predicted by the bimodal distribution is $\sim 1 \%$ for the number of the crystals produced. The observation suggests that the formation of crystals is likely due to heterogeneous nucleation. The chirality of the crystals may somehow be influenced by the impurities already existing in the solution or coming from air. This conjecture seems to be supported by a set of independent measurements where carefully filtered, concentrated sodium chlorate solutions were flame sealed in ampules. These samples can be supercooled by as much as $100^{\circ}$ without crystalization!

2. Nucleation in Stirred Solutions

To have a "quality control" to the experiment, we simultaneously run quiescent samples (typically four) together with the stirred samples. If these reference samples showed notable bias in the chirality of the crystals, which rarely occurred due to contamination, the entire run is discarded.

Systematic measurements were carried out for samples containing $V=5$ and $7.5 \mathrm{cc}$ of solutions. For samples with $V=5 \mathrm{cc}$, the magnetic bars touch the bottom of the dishes when the nucleation occurs. whereas for samples with $V=7.5 \mathrm{cc}$, the magnetic bars always stay afloat. 
Mechanical stirring has a dramatic effect on the population of two chiral species for samples containing small amount of solutions $(V=5 \mathrm{cc})$. In this case we found that for $f>>1 \mathrm{~Hz}$, each sample is populated either by the left or by the right-handed crystals, yielding high chiral purity with a great certainty. The situation is different at low speeds, $0<f \leq 1 \mathrm{~Hz}$. The degree of chiral symmetry breaking, in this case, depends on the rotation speed of the magnetic mixer. Figure 2 shows a series measurements with different $f$, ranging from $0.02 \mathrm{hz}$ to $1 \mathrm{hz}$. For each stirring frequency, twenty samples were examined. The total number of crystal counts are of the order of 10,000 for the high frequency end and of the order of 1,000 for the low frequency end. We note that even at very low frequencies, such as $f \sim 0.02 \mathrm{hz}$, the probability distribution function, PDF, is already significantly different from that of a quiescent solution as shown in Fig. 1. The broadening of the central peak indicates the chiral symmetry in these samples is partially broken. As the stirring rate increases, the PDF starts to peak around the two chirally pure state with $O= \pm 1$. For instance, at $f=1 \mathrm{hz}$, among the twenty samples, eight of them are left-handed with $O \sim+0.99$, twelve samples are right-handed with an average order $O \simeq-0.95$.

To characterize the order of an assemble of samples by considering $O>0$ and $O<0$ to be degenerate, we define an ensemble averaged order parameter: $O_{a v g}=\left[\sum_{O} O^{2} P(O) / \sum_{O} P(O)\right]^{1 / 2}$. By this definition we understand that $O_{a v g}$ is non-zero even for runs with no stirring. According to Fig. 1 , the finite width in the PDF gives $O_{\text {avg }} \simeq 25 \%$. Figure 3 shows $O_{\text {avg }}$ as a function of stirring frequency $f$ ranging from 0.01 to $3 \mathrm{hz}$. Here the data are plotted as solid squares. As can be seen, for $f>1$ hz the samples are almost completely ordered with optical purity approaching nearly $100 \%$, while for $f<1$ hz only partial ordering were observed. The variation of $O_{\text {avg }}$ vs. $f$ appears to be smooth, and for all the runs $O_{a v g}$ is significantly higher than the background level of $25 \%$. The large statistical noise seen in the data, particularly for the partially ordered samples, is also interesting, and it reflects the fact that the distribution function is extremely broad in the range between $-1<0<1$.

An interesting observation, which was caused by an unintended event, was that the chiral ordering reduced notably for samples containing slightly larger volumes of solution, while the mixing condition remained the same. We pursued the matter systematically. Fig. 4 shows the PDF for samples containing $7.5 \mathrm{cc}$ solution. The stirring frequency is varied between 0.01 to $4.0 \mathrm{hz}$. The distribution is in remarkable contrast with runs having a small sample volume, see Fig. 2. A fair comparison is perhaps for $f=1 \mathrm{hz}$. Here we observed that the PDF is essentially flat with little or no enhancement at $O= \pm 1$. To convince ourselves that the effect we were observing was real, we repeated the measurement many times. Instead of using 20 samples, in this set of measurements $(f=1 \mathrm{hz}) 92$ samples were used. Increasing the stirring frequency to 2 or $4 \mathrm{hz}$ does not change the PDF drastically, and the chiral symmetry is only weakly broken. In Fig. 3 we also show $O_{\text {avg }}$ vs. $f$ for samples with $V=7.5 \mathrm{cc}$. As shown by open circles, here we find that the data has only a weak frequency dependence, and the overall value of the order is not much greater than the background value of $25 \%$. We note that if PDF is a flat distribution, $O_{\text {avy }}$ is approximately $60 \%$, which is comparable to what we observed at high stirring frequencies. At best, the chiral symmetry is only weakly broken in this case.

\section{Nucleation in Electro-convection}

The nucleation experiment was also carried out in an electro-convection cell with different flow characteristics. For low currents, the vortices are localized in space and form a fairly regular lattice. For a high current, however, the vortices become delocalized and the flow is nearly turbulent. The local velocity in the electro-convection cell can be a few $\mathrm{cm} / \mathrm{s}$ which is at least comparable to the mechanical stirring experiments. It is anticipated, therefore, that if hydrodynamic flow is responsible for the symmetry breaking, we would see localized patches of crystals with broken symmetry. In the strong flow regime, a single chiral species may even dominate the entire population in the cell. This is not what has been observed experimentally. For all different flow rates, no strong evidence of chiral symmetry breaking was observed in this set of experiments.

\section{INTERPRETATION}

It should be noted that in our experiments, both with and without stirring, the initial nucleation almost always occurs on the liquid-vapor interface. Typically one sees several crystals float on the surface, and their chirality is not dominated by either handedness. As the crystals grow to a fraction of a millimeter. they fall to the bottom due to gravity. The surface nucleation itself is not surprising in that it is well known that impurities and surfaces can significantly lower the energy barrier of nucleation. However, surface nucleation can explain the unexpected volume dependence seen in the experiment. For a small volume, the magnetic stirring bar makes contact with the bottom of the dish. A settled crystal can be caught between the surfaces of the mixer and the dish, and can be crushed. A single catastrophic event like this, if it could produce a large number of small crystals, can severely bias the population since the initial number of the crystals is very small due to a large activation energy for nucleation, $\sim 100 k_{B} T$. For a large volume, there still is a small amount of mechanical contact between the mixer and the crystals on the liquid surface, but the crushing of settled crystals between the mixer and dish is practically eliminated. 
Quantitatively it is not difficult to show that hydrodynamic interaction alone is not sufficient to cause a rapid production of the secondary nuclei. To see this we estimate the stress on a nucleus due to hydrodynamic shear, $\sigma=\eta \gamma$, where $\eta(=0.1$ poise $)$ is the shear viscosity of the solution and $\gamma$ is the shear rate. In our experiment $\gamma \sim f \sim 1 \mathrm{hz}$, we found $\sigma \sim 10^{-1} \mathrm{erg} / \mathrm{cm}^{3}$. This is very small compared to the energy density $\epsilon$ of a fully-grown crystal, $\epsilon=k_{B} T \rho \sim 10^{9} \mathrm{erg} / \mathrm{cm}^{3}$ for $\rho \sim 10^{23} \mathrm{~cm}^{-3}$. However, it is easy to show that stress produced by the weight of the magnetic bar $\sigma\left[\equiv \mathrm{mg} / \mathrm{a}^{2}\right] \simeq 10^{6}$ is not insignificant compared to $\epsilon$. Here $m=0.1 \mathrm{~g}$ is the weight of the mixer, $g \simeq 1000 \mathrm{~cm} / \mathrm{s}^{2}$ is the gravitational acceleration, and $a=0.01 \mathrm{~cm}$ is the size of the crystal. We note that $\epsilon$ estimated above is for a bulk crystal material, which may not be applicable to micro-crystals, or to the edges and the corners of a fully-grown crystals. The mechanical strength of those parts can be significantly lower than $\epsilon$, and they are prone to mechanical damage by either the weight or the impact of the magnetic mixer. Indeed, a close inspection of crystals for some our samples reveals clear signs of mechanical damage. Unlike those crystals grown from a quiescent solution, under stirring conditions, the shape of certain crystals is highly irregular, with missing corners and rounded edges. The null result observed in our electro-convection experiment is also consistent with our interpretation.

The effects of mechanical damage on the chiral symmetry breaking of $\mathrm{NaClO}_{3}$ crystals can be mimicked by the following simple growth model. A difference equation for the growth of each crystal species can be formulated where in each time step, one crystal is nucleated from the solution. This crystal will be either left or right handed. In addition to nucleation, crystals can be created by crushing. If we denote the crushing rate by $\lambda$, the the population of right handed, $N^{R}$, and left handed, $N^{L}$, crystals at any time step, $i$, is given by:

$$
\begin{gathered}
N_{i+1}^{R}=N_{i}^{R}+k+\lambda N_{i}^{R} \\
N_{i+1}^{L}=N_{i+1}^{L}+! k+\lambda N_{i}^{L},
\end{gathered}
$$

where $i=1 \ldots N_{\max }$. Here $k$ is randomly chosen to be either 0 or 1 per step, and $! k$ is the opposite of $k$. That is, if $N^{R}$ grows one crystal, $N^{L}$ does not grow one. The iteration continues until the solution is depleted (i.e. $\left.i=N_{\max }\right)$. It is obvious that this scheme produces a binomial distribution when $\lambda=0$. Figure 5 shows the symmetry breaking when $\lambda$ is varied from 0 to 10 . For each value of $\lambda$ the PDF is plotted against the order parameter $O$. All distribution functions were calculated using 100 independent runs, and each run evolved for $N_{\max }=1000$ steps.

Despite its simplicity, with no hydrodynamics or thermodynamics, our model nonetheless captures some basic features seen in the experiment. Namely, in the absence of the mechanical damage $\lambda=0$, the PDF is strongly peaked at $O=0$. The introduction of a small crushing parameter immediately broadens the distribution function. As the crushing parameter increases, the distribution shifts continuously towards the states with $O= \pm 1$, in a similar fashion as our experiments with small samples.

\section{CONCLUSIONS}

In conclusion, using different flow. geometries we find that the chiral symmetry breaking in supersaturated $\mathrm{NaClO}_{3}$ solutions cannot be explained by hydrodynamic convection as suggested by a number of earlier experiments ${ }^{1,6}$ and theories ${ }^{2,3}$. The effect appears to be purely mechanical, resulting from mixercrystal interaction. In effect, the phenomenon is not much different from seeding the solution with numerous micro-crystals created from a single "mother" crystal. This mechanism of generating secondary nuclei was previous considered by Kondepudi and Sabanayagam ${ }^{\text {? }}$. However, its severity and extent have not been fully appreciated. We emphasize that by eliminating mechanical contacts with the precipitated crystals, even in the presence of a chaotic flow, there will be no chiral symmetry breaking. Using a simple growth model, we demonstrate that the phenomenon is essentially controlled by a single parameter, the rate of crushing $\lambda$. By varying $\lambda$, the system can be in either non-chiral, weakly chiral, or strongly chiral symmetry breaking state. The PDF generated from our simple model is also in good agreement with our measurements.

\section{ACKNOWLEDGMENTS}

We would like to thank W.I. Goldburg and C. Yeung for many helpful discussions during the course of this research. This research is supported by NASA under grant No. NAG8-959. 


\section{REFERENCES}

${ }^{1} D$. Kondepudi, R. Kaufman, and N. Singh, Science 250, 975 (1990).

${ }^{2}$ Guy Metcalfe and J.M. Ottino, Phys. Rev. Lett. 72, 2875 (1994).

${ }^{3}$ Irving R. Epstein, Nature 374, 321 (1995).

${ }^{4}$ The sodium chlorate, $\mathrm{NaClO}_{3}$, was purchased from Fisher Scientific. The chemical is $99.9 \%$ pure and was used without further purification.

${ }^{3}$ F.S. Kipping and W.J. Pope, J. Chem. Soc. Trans. 73, 606 (1898).

${ }^{6} \mathrm{X}-\mathrm{l}$. Wu, B. Martin, and A. Tharrington, Proceeding of "Second Microgravity Fluid Physics Conference," 319 (1994).

${ }^{7}$ D.K. Kondepudi and C. Sabanayagam. Chem. Phys. Lett. 217, 364 (1994).

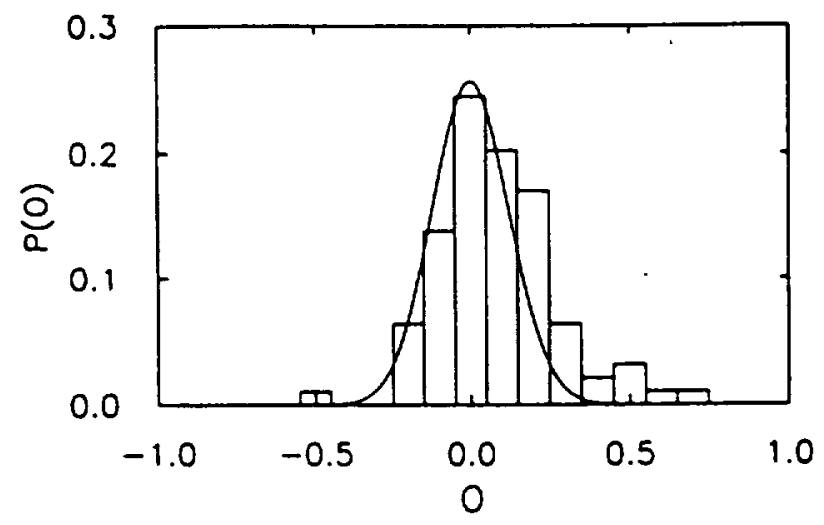

Figure 1. The order-parameter probability distribution function $P(O)$ for quiescent samples.

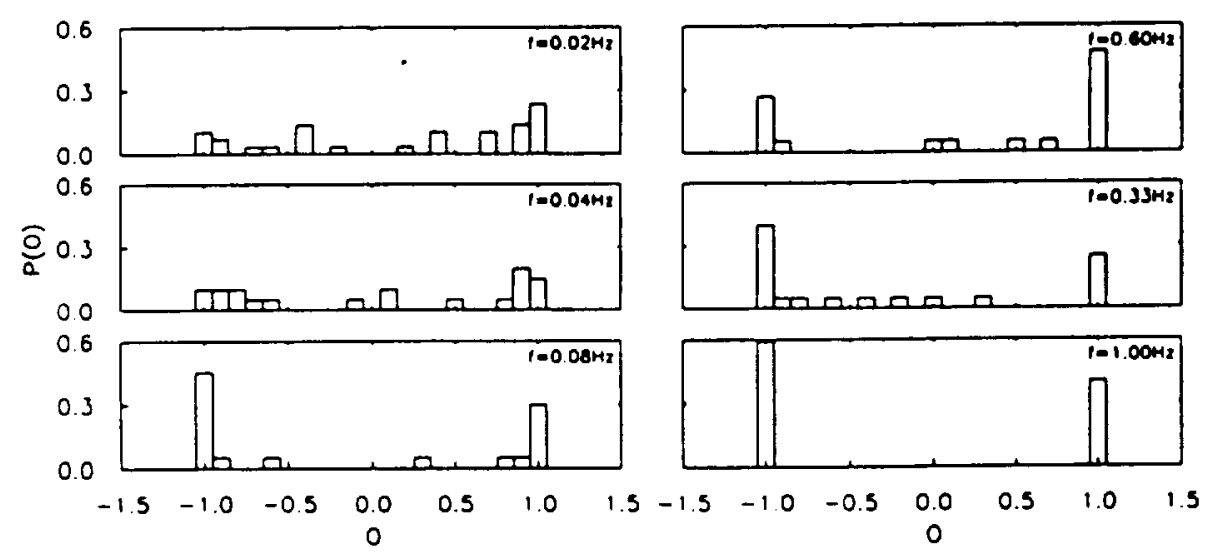

Figure 2. The order parameter probability distribution functions with different stirring rates. The volume of the solution is $V=5.0 \mathrm{cc}$. 


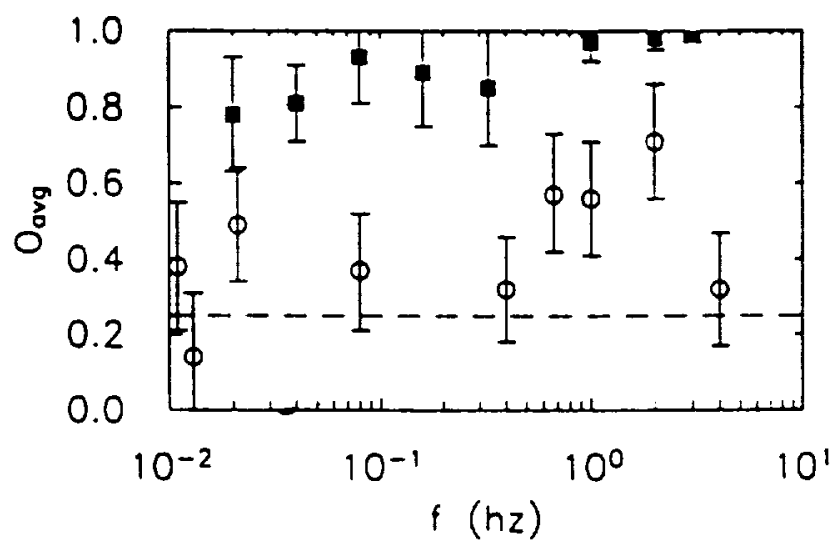

Figure 3. The solid squares are for samples with $V=5.0 \mathrm{cc}$ and the open circles are for samples with $V=7.5 \mathrm{cc}$. The error bars are due to statistical errors. The dashed line is the background, see text for details.

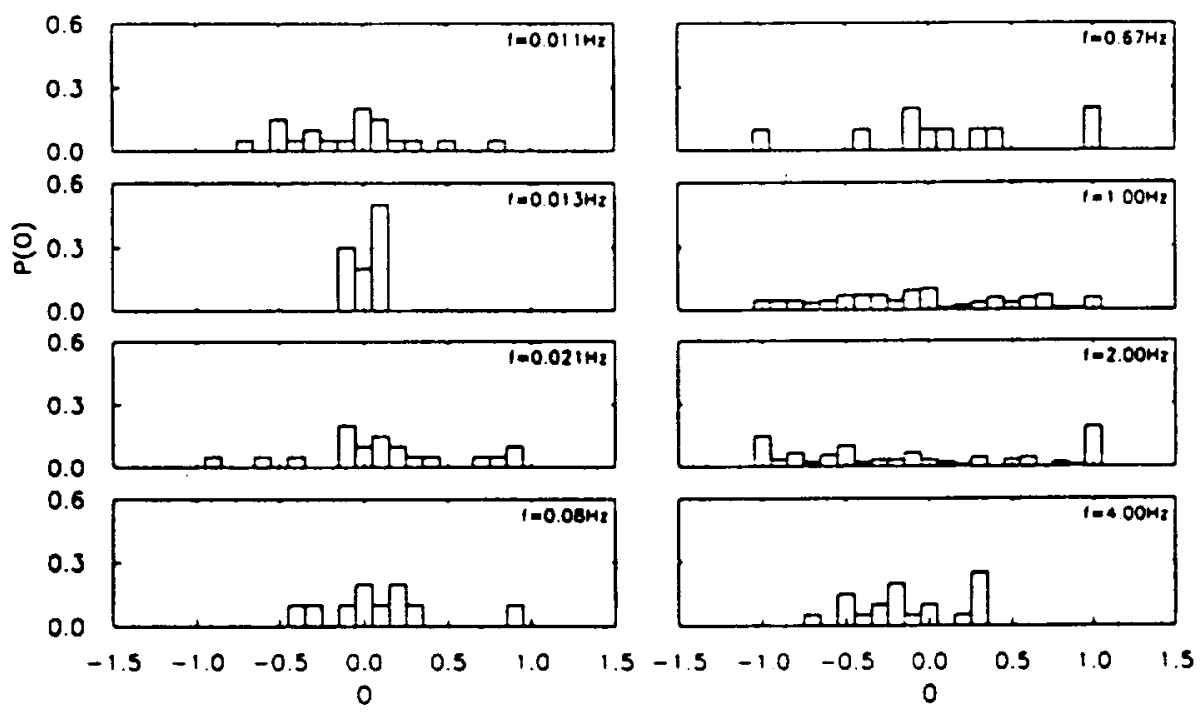

Figure 4. The order parameter probability distribution functions with different stirring rates. The volume of the solution is $V=7.5 \mathrm{cc}$.

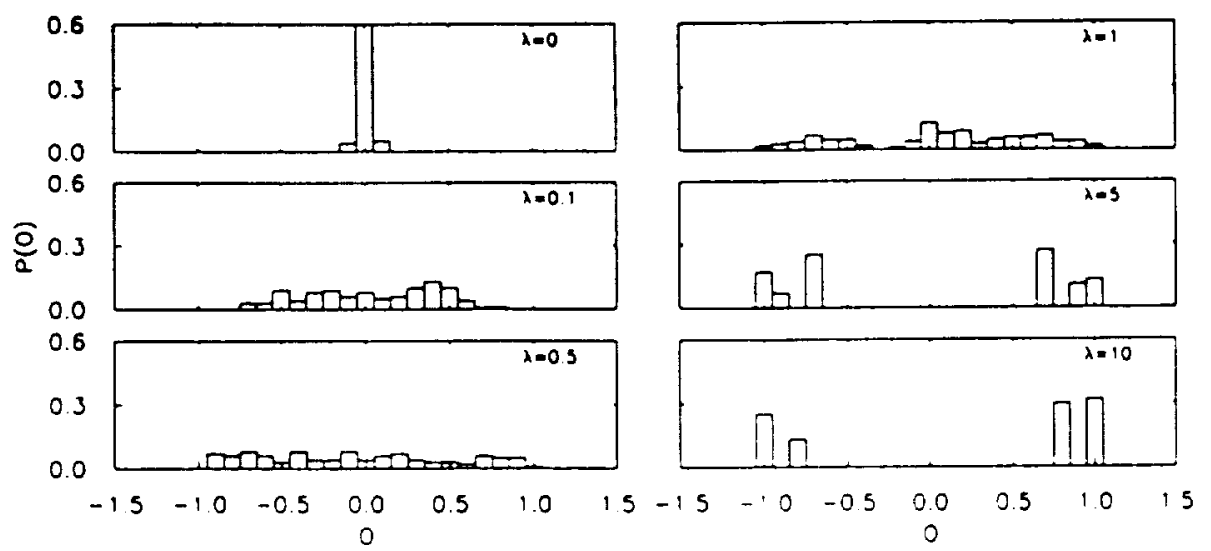

Figure 5. Model calculations for probability distribution functions with different crushing rates. 